\title{
NUMERICAL METHODS FOR SOLVING "RIGID SYSTEMS" APPLIED FOR THE PROBLEM OF THE DYNAMICS OF HEAVY-LOADED BEARINGS
}

\author{
Yu. V. Rozhdestvensky ${ }^{1}$, ruv@susu.ac.ru \\ E. A. Zadorozhnaya ${ }^{1}$, elena-nea@rambler.ru \\ R. O. Chuchelov ${ }^{1}$, chro_174@mail.ru \\ D. I. Raskatov ${ }^{1}$, dir.77@mail.ru \\ ${ }^{1}$ South Ural State University, Chelyabinsk, Russian Federation
}

The problem of the dynamics of fluid friction bearings was formulated in the article. For "rigid system" the classical methods of integration of the system of differential equation of movement proved to be unsuitable because of the need to reduce the next step of integration in some parts of the journal center trajectory to very small values. We have described the main approaches that used for solving the problem. We also presented the main methods that have proven themselves in the solution of problems of the dynamics of rapidly rotating rotors and autonomous bearings of heat engines. Numerical methods for "rigid system"and algorithms for solving the problems of the dynamics were given. With the help of numerical experiments coefficients of the equations was performed. The step for calculating adjusted to the minimum value of the discrepancy.).

Keywords: heavy-loaded bearings, the problem of dynamics, "rigid system methods of prediction-correction, order of convergence.

\section{Introduction}

The movement of the journal on the lubricating layer in heavy-loaded bearing of fluid friction as a free rigid body can be represented by a set of two of his movements: forward together with the mass center of the journal in the inertial coordinate system and the spherical around the center of mass. The problem of the dynamics of fluid friction bearings in this case reduces to solving a system of approximate equations written in matrix form:

$$
\begin{aligned}
& m \ddot{u}=F(t)-P(u, \dot{u}, \gamma, \dot{\gamma}) \\
& J \ddot{\gamma}=M_{a}(t)-M_{r}(u, \dot{u}, \gamma, \dot{\gamma})+M_{G},
\end{aligned}
$$

where $u, \gamma$ - the displacement vector of the journal center and angular movements of the journal in the inertial space; $m, J$ - the parameters which characterize the mass and moment of inertia of the journal; $F(t), M_{a}(t)$ - active force and moment couple of forces acting on the journal; $P, M_{r}$ - the force and moment acting on the bearing by the lubricant layer; $M_{G}$ - the gyroscopic moment.

The system of equations (1) to the journal, rotating at an angle in the bearing, can be written as

$$
\begin{aligned}
& m \ddot{u}+P(u, \dot{u}, \gamma, \dot{\gamma})=f(\tau) ; \\
& J \ddot{\gamma}+M_{r}(u, \ddot{u}, \gamma, \ddot{\gamma})=\bar{M}_{a}(\tau)+\bar{M}_{G}(\tau)=\bar{M}(\tau),
\end{aligned}
$$

where

$$
u=\left[\begin{array}{c}
x \\
y
\end{array}\right] ; \quad P=-\frac{k_{F}}{h_{0} \omega_{0}^{2}}\left[\begin{array}{l}
\bar{R}_{x}(u, \dot{u}, \gamma, \dot{\gamma}) \\
\bar{R}_{y}(u, \dot{u}, \gamma, \dot{\gamma})
\end{array}\right] ; \quad f=\left[\begin{array}{c}
f_{x}(\tau) \\
f_{y}(\tau)
\end{array}\right]
$$




$$
\gamma=\left[\begin{array}{c}
\alpha^{\prime} \\
\beta^{\prime}
\end{array}\right] ; \quad M_{r}=-\frac{k_{M}}{\omega_{0}^{2}}\left[\begin{array}{c}
\bar{M}_{r x}(u, \dot{u}, \gamma, \dot{\gamma}) \\
\bar{M}_{r y}(u, \dot{u}, \gamma, \dot{\gamma})
\end{array}\right] ; \quad \bar{M}(\tau)=\left[\begin{array}{c}
\bar{M}_{a x}(\tau)+\bar{M}_{G x}(\tau) \\
\bar{M}_{a y}(\tau)+\bar{M}_{G y}(\tau)
\end{array}\right],
$$

$u=u / h_{0} ; x, y, \alpha^{\prime}, \beta^{\prime}$ - dimensionless coordinates of the journal in the inertial space; $\bar{R}_{x}$, $\bar{R}_{y}, \bar{M}_{r x}, \bar{M}_{r y}$ - dimensionless reaction of the lubricant layer determined from the Reynolds equation; $m, J$ - mass and moment of inertia of the journal; $f_{x}(\tau), f_{y}(\tau), \bar{M}_{a x}(\tau)$, $\bar{M}_{a y}(\tau)$ - external forces and moments; $\bar{M}_{G x}(\tau), \bar{M}_{G y}(\tau)$ - gyroscopic moments; $\tau=\omega_{0} t$ - the dimensionless time; $k_{F}, k_{M}$ - coefficients for transfer to dimensionless quantities forces and moments; $f(\tau)=F(t) / \omega_{0}^{2} h_{0} ; \bar{M}(\tau)=M(t) / \omega_{0}^{2} ; k_{F}=B D \mu_{0} \omega_{0} / \psi^{2}$; $k_{M}=B D^{2} \mu_{0} \omega_{0} / 2 \psi ; \psi=h_{0} / R, R$ - the radius of the journal.

In the so-called massless models, when the forces of inertia due to the motion of the stud on a thin lubricating layer have the second order of smallness in comparison with the loads $F(t), M_{a}(t)$, the equation of motion (1) is replaced by the equilibrium equations:

$$
\begin{aligned}
& 0=F(t)-P(u, \dot{u}, \gamma, \dot{\gamma}) \\
& 0=M_{a}(t)-M_{r}(u, \dot{u}, \gamma, \dot{\gamma})+M_{G} .
\end{aligned}
$$

Methods for solving problems of the dynamics of the journal on the lubricating layer in the bearing depends primarily on the nature of stresses. For bearing of piston engines the system of motion equations are usually considered in the form of the equilibrium equations (3), neglecting the weight force of the stud. In considering the tasks of stability of rotors it is in the form of Newton (2). In this case, a stability of the balanced journal is often examined by linearization of the motion equations [1,2]. This approach allows us to accurately determine the critical speed at which the equilibrium position of the journal becomes unstable, but does not provide information about the nature of the motion path of the stud. Knowledge of the trajectory (orbit) of the journal motion loaded with inertial forces from the unbalanced mass need to assess its orbital stability. Therefore, only the solution to the problem in the nonlinear formulation makes it possible to calculate and consider trajectory and journal oscillations with amplitudes that are commensurate with the magnitude of the gap between the journal and the bearing $[3,4]$.

\section{The main approaches to solving problems of dynamics}

If we perform the discretization of the motion equations system (2) with respect to time, the decision in going to the next step in time can be obtained using the explicit or implicit method of calculation. In the explicit scheme unknowns are the coordinates of the journal center and pressure, and in the implicit scheme - the pressure and rate of change of the journal position. In [4], these methods have been studied in detail, and preference was given to the implicit scheme, since in the explicit scheme there is a tendency to accumulation of rounding errors in repeated arithmetic operations.

A common way to solve the system (2) is to reduce it to a first order differential equation

$$
\left\{\begin{array}{l}
\dot{u}=\nu \\
m \dot{\nu}+P(u, v, \gamma, v)=f(\tau) \\
\dot{\gamma}=v \\
J \dot{v}+M_{r}(u, v, \gamma, v)=\bar{M}(\tau)
\end{array}\right.
$$

and application of known algorithms for solving the Cauchy task. Numerical integration of first order differential equations solved with respect to derivatives, can be performed by 
many well-known methods, which by the nature of the techniques used at each integration step, were divided into one-step and multi-steps. The most widely used in practice the Runge-Kutta method, belonging to the group of single-step, and methods of prediction -correction, which are multi-steps.

Methods of prediction-correction are based on the use of finite difference expressions of the form

$$
u_{n}=\sum_{i=1}^{k} \alpha_{i} u_{n-1}+\Delta t \sum_{i=0}^{k} \beta_{i} f_{n-i},
$$

where $k$ - the order of method; $\Delta t$ - the time step; $n$ - the number of time step; $\alpha_{i}$ and $\beta_{i}$ - constant coefficients determined from the condition and accuracy of the method.

In the explicit Runge-Kutta methods the system of equations (4), written in the form

$$
\dot{U}_{n}=F\left(U_{n}, \tau_{n}\right)
$$

and solved by using the following basic formula:

$$
U_{n+1}=U_{n}+\Delta U_{n}
$$

Here $U=[u, \dot{u}, \gamma, \dot{\gamma}]^{T}$. A method for calculating the function increment $\Delta U_{n}$ depends on the order of the method.

In the explicit Runge-Kutta methods and prediction-correction for sustainability the next relation must satisfy:

$$
\Delta t_{\max } \cdot\left|\operatorname{Re} \lambda_{i}\right| \leq C, \quad C=\text { const }
$$

where Re $\lambda_{i}$ - the real parts of the eigenvalues $\lambda_{i}$ of the Jacobi matrix formed at each step of the integration from the right-hand side of (4).

These methods have worked well for solving the dynamics of rapidly rotating rotors on bearings with intermediate elements in the form of rotating and non-rotating floating bushings. The result of the solving, in addition to the trajectory of the journal center and the spatial position of the axis of rotation of the journal in a bearing, is a set of output parameters characterizing resistance, wear resistance, the heat-load, fatigue life support, etc $[5,6]$.

The experience with these methods has shown that for some systems, known as "rigid" mentioned methods are not applicable [7].

Systems in the range $J \subset[a, b]$ can be considered as "rigid" system, if for $\tau \in J$

$$
\left.\begin{array}{l}
\operatorname{Re}\left(\lambda_{k}\right)<0, k=1,2, \ldots S \\
S(\tau)=\max \operatorname{Re}\left(-\lambda_{k}\right) / \min \operatorname{Re}\left(-\lambda_{k}\right)>>1, k=1,2, \ldots S ;
\end{array}\right\}
$$

where Re $\lambda_{k}$ - the real part of the eigen values $\lambda_{k}$ of the Jacobi matrix of the system, which is obtained by linearization of a nonlinear system of differential equations at a time $\tau$.

Conditions (5) indicate that for the "rigid" system the wide scatter of the eigen values of the Jacobi matrix is typical. In some test problems discussed by the authors, the difference in eigen values of the Jacobi matrix of the linearized system (4) was several orders of magnitude. In such cases, the classical methods of integration of the system (4) proved to be unsuitable because of the need to reduce the next step of integration in some 
parts of the journal center trajectory to very small values. In this case, on the interval of the local stiffness of the system (4) the number of integration steps is comparable to the ratio $S(\tau)$.

The phenomenon of "rigidity" effected on the speed of the transition process in the journal-bearing system. This is especially important in problems of stability of an unbalanced rotor, as well as in the analysis of the dynamics of the bearing of piston engines, which are characterized by rapid changes in vector-valued function $f(\tau)$.

The range of variation of the integration step is greatly extended by using special techniques, focused on solving the "rigid" systems of equations, for which large differences in the eigen values of the Jacobi matrix are characterized. These include local linearization method [8], Fowler-Wharton [9], implicit one-step and multi-step methods, and others.

\section{Numerical methods for solving rigid systems}

For "rigid" systems because of the very small time step and the instability of traditional methods such as Runge-Kutta method, one of the way of solution this problem was the use the implicit numerical methods. The main methods for solving the equations of motion of the journal on the lubricating layer are considered below. They are designed with reference to problems of nonlinear dynamics bearings piston- and rotary machines.

In the method of Fowler-Wharton the displacement vector at a time $\tau_{n+1}=\tau_{n}+\Delta \tau$ determined as

$$
u_{n+1}=u_{n}+\Delta \tau \dot{u}_{n-1}+\Delta \tau c_{1} d_{1}
$$

where

$$
\begin{gathered}
\dot{u}_{n-1}=\left(u_{n}-u_{n-1}\right) / \Delta \tau_{1} ; \quad c_{1}=\left\{\begin{array}{l}
\left(e^{\lambda \Delta \tau}-1\right) / \lambda \Delta \tau, \lambda<0 \\
1+\lambda \Delta \tau / 2, \lambda \geq 0
\end{array} ; \quad \lambda=\left\{\begin{array}{l}
\ddot{u}_{n} / d_{1}, d_{1} \neq 0 \\
0, d_{1}=0
\end{array} ;\right.\right. \\
d_{1}=\dot{u}_{n}-\dot{u}_{n-1} ; \ddot{u}_{n}=\dot{u}_{p}-\dot{u}_{n} / \Delta \tau ; \\
\dot{u}_{p}=F\left[\tau_{n}+\Delta \tau, u_{p}\left(\tau_{n}+\Delta \tau\right)\right] ; \quad u_{p}(\tau+\Delta \tau)=u_{n}+\Delta \tau \dot{u}_{n} ; \\
\Delta \tau \leq \Delta \tau / 4 ; \quad \Delta \tau=\tau_{n+1}-\tau_{n} ; \quad \Delta \tau_{1}=\tau_{n}-\tau_{n-1} .
\end{gathered}
$$

At the beginning we take into account $u_{n}=u_{n-1}$. The value $\Delta \tau$ is controlled by calculating the local integration errors for each of variables.

$$
E_{n+1}=\left|\Delta t\left[\dot{u}_{n+1}-\left(\dot{u}_{n-1}+c_{0} d_{1}\right)\right]\right|,
$$

where

$$
c_{0}= \begin{cases}e^{\lambda \Delta \tau}, & \text { if } \quad \lambda<0 \\ 1+\lambda \Delta \tau, & \text { if } \quad \lambda \geq 0 .\end{cases}
$$

In the process of integrating the motion equations of the journal to adjust the time step we used following algorithm.

Upper and lower limits for the admissible errors were calculated:

$$
A_{u, n+1}=a_{1}+a_{2}\left|u_{n+1}\right|, \quad B_{u, n+1}=b_{1}+b_{2}\left|u_{n+1}\right|
$$

where coefficients were determined by numerical experiments $a_{1}=0.0005 ; a_{2}=0.0075$; $b_{1}=0.00001, b_{2}=0.00005$.

Then the following four situations were analyzed: 
a) if for some values $u$ the relation $E_{u, n+1} / A_{u, n+1}>1,5$ is true, the integration step was divided in half, and the result of integration is not taken;

b) if $E_{u, n+1} / A_{u, n+1}>0,75$ for some values $u$ and $E_{u, n+1} / A_{u, n+1} \leq 1,5$ for all $u$, the value of the current integration step was taken, but for subsequent calculations the step was divided in half;

c) if $E_{u, n+1} / A_{u, n+1} \leq 0,75$ and $E_{u, n+1} / B_{u, n+1} \geq 1$ for all $u$, step has't changed;

d) if $E_{u, n+1} / A_{u, n+1} \leq 0,75$ for all $u$ and $E_{u, n+1} / B_{u, n+1}<1$ at least one $u$, integration step is doubled.

\section{The modified method of the linear acceleration (Wilson method)}

In [4] the method of local linearization of the system of equations (1) considered in relation to tasks of rotors stability. Its application in practice has shown that it does not satisfy the requirements of universality and, in many cases, when in the left side of equation (1) the coefficient of the highest derivative is small, does not provide a solution. In connection with this, the Wilson's method [8] seems to be preferable. Its can be regarded as a modified method of local linearization (linear acceleration method). The essence of the method, we consider on the example of the solution of the first equation of system (1).

Linearize the equation on a short time interval

$$
m \Delta \ddot{u}(\vartheta \Delta \tau)+C(\tau) \Delta \dot{u}(\vartheta \Delta \tau)+K(\tau) \Delta u(\vartheta \Delta \tau)=\Delta f(\vartheta \Delta \tau) .
$$

Here $\Delta u=\left[\begin{array}{c}\Delta x \\ \Delta y\end{array}\right]$ - the vector of increments; $C(\tau)=-\frac{k_{F}}{h_{0} \omega_{0}^{2}} \frac{\partial \bar{R}}{\partial \dot{u}}-$ the damping matrix; $K(\tau)=-\frac{k_{F}}{h_{0} \omega_{0}^{2}} \frac{\partial \bar{R}}{\partial u}-$ the stiffness matrix; $\bar{R}-$ dimensionless reaction of the lubricant layer;

$$
\frac{\partial \bar{R}}{\partial \dot{u}}=\left[\begin{array}{cc}
\frac{\partial \bar{R}_{x}}{\partial \dot{x}} & \frac{\partial \bar{R}_{x}}{\partial \dot{y}} \\
\frac{\partial \bar{R}_{y}}{\partial \dot{x}} & \frac{\partial \bar{R}_{y}}{\partial \dot{y}}
\end{array}\right] ; \quad \frac{\partial \bar{R}}{\partial u}=\left[\begin{array}{cc}
\frac{\partial \bar{R}_{x}}{\partial x} & \frac{\partial \bar{R}_{x}}{\partial y} \\
\frac{\partial \bar{R}_{y}}{\partial x} & \frac{\partial \bar{R}_{y}}{\partial y}
\end{array}\right]
$$

Wilson's method assumes a linear change in acceleration in the extended range $\tau$, $\tau+\vartheta \Delta \tau$, where $\vartheta \geq 1$. If $\vartheta=1$, this is the usual version of the method of linear acceleration (local linearization).

Taking a linear dependence of the components of the acceleration vector

$$
\ddot{u}(\tau+\Delta \tau)=\ddot{u}(\tau)+\frac{\Delta \tau}{\vartheta \Delta \tau}(\ddot{u}(\tau+\vartheta \Delta \tau)-\ddot{u}(\tau)) .
$$

and integrating, we obtain

$$
\begin{gathered}
\dot{u}(\tau+\Delta \tau)=\dot{u}(\tau)+\ddot{u}(\tau) \Delta \tau+\frac{\Delta \tau^{2}}{2 \vartheta \Delta \tau}(\ddot{u}(\tau+\vartheta \Delta \tau)-\ddot{u}(\tau)) ; \\
u(\tau+\Delta \tau)=u(\tau)+\dot{u}(\tau) \Delta \tau+\frac{\Delta \tau^{2}}{2} \ddot{u}(\tau)+\frac{\Delta \tau^{3}}{6 \vartheta \Delta \tau}(\ddot{u}(\tau+\vartheta \Delta \tau)-\ddot{u}(\tau)) .
\end{gathered}
$$


Using an increment $\Delta u$ as the main variable, taking into account the equations (8) and (9) we find for the end of the interval:

$$
\begin{aligned}
& \Delta \ddot{u}(\vartheta \Delta \tau)=\frac{6}{\vartheta^{2} \Delta \tau^{2}} \Delta u(\vartheta \Delta \tau)-\frac{6}{\vartheta \Delta \tau} \dot{u}(\tau)-3 \ddot{u}(\tau) \\
& \Delta \dot{u}(\vartheta \Delta \tau)=\frac{3}{\vartheta \Delta \tau} \Delta u(\vartheta \Delta \tau)-3 \dot{u}(\tau)-\frac{\vartheta \Delta \tau}{2} \ddot{u}(\tau)
\end{aligned}
$$

After substituting the expressions for $\Delta \ddot{u}$ and $\Delta \dot{u}$ in equation (6) we get

$$
\begin{gathered}
{\left[\frac{6 m}{\vartheta^{2} \Delta \tau^{2}}+C(\tau) \frac{3}{\vartheta \Delta \tau}+K(\tau)\right] \Delta u(\vartheta \Delta \tau)=m\left[\frac{6}{\vartheta \Delta \tau} \dot{u}(\tau)+3 \ddot{u}(\tau)\right]+} \\
+C(\tau)\left[3 \dot{u}(\tau)+\frac{\vartheta T}{2} \ddot{u}(\tau)\right]+\Delta f(\vartheta \Delta \tau)
\end{gathered}
$$

For infinitesimal dynamic movements of the journal center near the position corresponding to the start time interval $\tau \Delta x=d(\chi \cos \delta) ; \Delta y=d(\chi \sin \delta)(\chi$ - relative eccentricity; $\delta$ - angle determining the position of the center line)

$$
\Delta \dot{x}=\Delta \dot{\chi} \cos \delta-\chi \Delta \dot{\delta} \sin \delta ; \quad \Delta \dot{y}=\Delta \dot{\chi} \sin \delta+\chi \Delta \dot{\delta} \cos \delta
$$

therefore,

$$
\Delta \dot{\chi}=\Delta \dot{x} \cos \delta+\Delta \dot{y} \sin \delta ; \quad \Delta \dot{\delta} \chi=\Delta \dot{y} \cos \delta-\Delta \dot{x} \sin \delta
$$

We have identified $\Phi=\left[\begin{array}{c}\Delta \chi \\ \Delta \delta\end{array}\right]$, and find $\dot{\Phi}=\left[\begin{array}{c}\Delta \dot{\chi} \\ \Delta \dot{\delta}\end{array}\right]=\left[\begin{array}{cc}\cos \delta & \sin \delta \\ -\frac{\sin \delta}{\chi} & \frac{\cos \delta}{\chi}\end{array}\right] \cdot\left[\begin{array}{c}\Delta \dot{x} \\ \Delta \dot{y}\end{array}\right]$, then

$$
K(\tau)=-\frac{k_{F}}{h_{0} \omega_{0}^{2}} \frac{\partial \bar{R}}{\partial \Phi}\left[\begin{array}{cc}
\cos \delta & \sin \delta \\
-\frac{\sin \delta}{\chi} & \frac{\cos \delta}{\chi}
\end{array}\right], \quad C(\tau)=-\frac{k_{F}}{h_{0} \omega_{0}^{2}} \frac{\partial \bar{R}}{\partial \dot{\Phi}}\left[\begin{array}{cc}
\cos \delta & \sin \delta \\
-\frac{\sin \delta}{\chi} & \frac{\cos \delta}{\chi}
\end{array}\right]
$$

where

$$
\frac{\partial \bar{R}}{\partial \Phi}=\left[\begin{array}{cc}
\frac{\partial \bar{R}_{x}}{\partial \chi} & \frac{\partial \bar{R}_{x}}{\partial \delta} \\
\frac{\partial \bar{R}_{y}}{\partial \chi} & \frac{\partial \bar{R}_{y}}{\partial \delta}
\end{array}\right] \quad \frac{\partial \bar{R}}{\partial \dot{\Phi}}=2\left[\begin{array}{cc}
\frac{\partial \bar{R}_{x}}{\partial E} & -\frac{\partial \bar{R}_{x}}{\partial G} \\
\frac{\partial \bar{R}_{y}}{\partial E} & -\frac{\partial \bar{R}_{y}}{\partial G}
\end{array}\right]
$$

Multiplying the matrix in (13), we obtain

$$
K(\tau)=\frac{1}{h_{0} \omega_{0}^{2}} a_{u}=\frac{1}{h_{0} \omega_{0}^{2}}\left[\begin{array}{ll}
a_{x x} & a_{x y} \\
a_{y x} & a_{y y}
\end{array}\right] ; \quad C(\tau)=\frac{1}{h_{0} \omega_{0}^{2}} b_{u}=\frac{1}{h_{0} \omega_{0}^{2}}\left[\begin{array}{ll}
b_{x x} & b_{x y} \\
b_{y x} & b_{y y}
\end{array}\right],
$$

where rigidity $a_{u}$ and damping $b_{u}$ coefficients are determined by the equations

$$
\begin{aligned}
& a_{x x}=-k_{F}\left(\frac{\partial \bar{R}_{x}}{\partial \chi} \cos \delta-\frac{1}{\chi} \frac{\partial \bar{R}_{x}}{\partial \delta} \sin \delta\right) ; \quad a_{x y}=-k_{F}\left(\frac{\partial \bar{R}_{x}}{\partial \chi} \sin \delta+\frac{1}{\chi} \frac{\partial \bar{R}_{x}}{\partial \delta} \cos \delta\right) ; \\
& a_{y x}=-k_{F}\left(\frac{\partial \bar{R}_{y}}{\partial \chi} \cos \delta-\frac{1}{\chi} \frac{\partial \bar{R}_{y}}{\partial \delta} \sin \delta\right) ; \quad a_{y y}=-k_{F}\left(\frac{\partial \bar{R}_{y}}{\partial \chi} \sin \delta+\frac{1}{\chi} \frac{\partial \bar{R}_{y}}{\partial \delta} \cos \delta\right) ;
\end{aligned}
$$




$$
\begin{aligned}
& b_{x x}=-2 k_{F}\left(\frac{\partial \bar{R}_{x}}{\partial E} \cos \delta+\frac{1}{\chi} \frac{\partial \bar{R}_{x}}{\partial G} \sin \delta\right) ; \quad b_{x y}=-2 k_{F}\left(\frac{\partial \bar{R}_{x}}{\partial E} \sin \delta-\frac{1}{\chi} \frac{\partial \bar{R}_{x}}{\partial G} \cos \delta\right) ; \\
& b_{y x}=-2 k_{F}\left(\frac{\partial \bar{R}_{y}}{\partial E} \cos \delta+\frac{1}{\chi} \frac{\partial \bar{R}_{y}}{\partial G} \sin \delta\right) ; \quad b_{y y}=-2 k_{F}\left(\frac{\partial \bar{R}_{y}}{\partial E} \sin \delta-\frac{1}{\chi} \frac{\partial \bar{R}_{y}}{\partial G} \cos \delta\right),
\end{aligned}
$$

where $\bar{R}_{x}=-(W \cos \delta-V \sin \delta) ; \bar{R}_{y}=-(W \sin \delta+V \cos \delta)$.

Solving the system (3), we obtain

$$
\begin{aligned}
& {\left[\frac{6 m h_{0} \omega_{0}^{2}}{\vartheta^{2} \Delta \tau^{2}}+\frac{3 b_{x x}}{\vartheta \Delta \tau}+a_{x x}\right] \Delta x+\left[\frac{3 b_{x y}}{\vartheta \Delta \tau}+a_{x x}\right] \Delta y=} \\
& =h_{0} \omega_{0}^{2} \Delta f_{x}(\vartheta \Delta \tau)+3 m h_{0} \omega_{0}^{2}\left[\frac{2}{\vartheta \Delta \tau} \dot{x}(\tau)+\ddot{x}(\tau)\right]+ \\
& +b_{x x}\left[3 \dot{x}(\tau)+\frac{\vartheta \Delta \tau}{2} \ddot{x}(\tau)\right]+b_{x y}\left[3 \dot{y}(\tau)+\frac{\vartheta \Delta \tau}{2} \ddot{y}(\tau)\right] ; \\
& {\left[\frac{3 b_{y x}}{\vartheta \Delta \tau}+a_{y x}\right] \Delta x+\left[\frac{6 m h_{0} \omega_{0}^{2}}{\vartheta^{2} \Delta \tau^{2}}+\frac{3 b_{y y}}{\vartheta \Delta \tau}+a_{y y}\right] \Delta y=} \\
& =h_{0} \omega_{0}^{2} \Delta f_{y}(\vartheta \Delta \tau)+3 m h_{0} \omega_{0}^{2}\left[\frac{2}{\vartheta \Delta \tau} \dot{y}(\tau)+\ddot{y}(\tau)\right]+ \\
& +b_{y x}\left[3 \dot{x}(\tau)+\frac{\vartheta \Delta \tau}{2} \ddot{x}(\tau)\right]+b_{y y}\left[3 \dot{y}(\tau)+\frac{\vartheta \Delta \tau}{2} \ddot{y}(\tau)\right] .
\end{aligned}
$$

Then $\Delta x$ and $\Delta y$ determined from the relations

$$
\Delta x=\frac{C_{1} B_{2}-C_{2} B_{1}}{A_{1} B_{2}-A_{2} B_{1}} ; \Delta y=\frac{C_{2} A_{1}-C_{1} A_{2}}{A_{1} B_{2}-A_{2} B_{1}}
$$

where

$$
\begin{gathered}
A_{1}=\frac{6 m h_{0} \omega_{0}^{2}}{\vartheta^{2} \Delta \tau^{2}}+\frac{3 b_{x x}}{\vartheta \Delta \tau}+a_{x x} ; \quad A_{2}=\frac{3 b_{y x}}{\vartheta \Delta \tau}+a_{y x} ; \\
B_{1}=\frac{3 b_{x y}}{\vartheta \Delta \tau}+a_{x x} ; \quad B_{2}=\frac{6 m h_{0} \omega_{0}^{2}}{\vartheta^{2} \Delta \tau^{2}}+\frac{3 b_{y y}}{\vartheta \Delta \tau}+a_{y y} ; \\
C_{1}=h_{0} \omega_{0}^{2} \Delta f_{x}(\vartheta \Delta \tau)+3 m h_{0} \omega_{0}^{2}\left[\frac{2}{\vartheta \Delta \tau} \dot{x}(\tau)+\ddot{x}(\tau)\right]+ \\
+b_{x x}\left[3 \dot{x}(\tau)+\frac{\vartheta \Delta \tau}{2} \ddot{x}(\tau)\right]+b_{x y}\left[3 \dot{y}(\tau)+\frac{\vartheta \Delta \tau}{2} \ddot{y}(\tau)\right] \\
C_{2}=h_{0} \omega_{0}^{2} \Delta f_{y}(\vartheta \Delta \tau)+3 m h_{0} \omega_{0}^{2}\left[\frac{2}{\vartheta \Delta \tau} \dot{y}(\tau)+\ddot{y}(\tau)\right]+ \\
+b_{y x}\left[3 \dot{x}(\tau)+\frac{\vartheta \Delta \tau}{2} \ddot{x}(\tau)\right]+b_{y y}\left[3 \dot{y}(\tau)+\frac{\vartheta \Delta \tau}{2} \ddot{y}(\tau)\right] .
\end{gathered}
$$

Accumulation of errors can be avoided as follows. Since the calculations are done using approximate formulas, then at the time $\tau$ instead of the expression

$$
m \ddot{u}(\tau)+R(u(\tau), \dot{u}(\tau))=f(\tau)
$$


the exact equality performed

$$
m \ddot{u}(\tau)+R(u(\tau), \dot{u}(\tau))=f(\tau)+N e(\tau),
$$

where $N e(\tau)$ - error, defined by discarding terms of second and higher order of smallness.

Given that at time $\tau+\vartheta \Delta \tau$

$$
m \ddot{u}(\tau+\vartheta \Delta \tau)+R(u(\tau+\vartheta \Delta \tau), \dot{u}(\tau+\vartheta \Delta \tau))=f(\tau+\vartheta \Delta \tau),
$$

equation (12) can be written as

$$
m \Delta \ddot{u}(\vartheta \Delta \tau)+\frac{\partial R}{\partial u} \Delta u(\vartheta \Delta \tau)+\frac{\partial R}{\partial \dot{u}} \Delta u(\vartheta \Delta \tau)=\Delta f(\vartheta \Delta \tau)-N e(\tau) .
$$

Substituting (10), (11) for $\Delta \dot{u}$ and $\Delta \ddot{u}$ in (8) find the equation

$$
\begin{gathered}
{\left[\frac{6 m}{\vartheta^{2} \Delta \tau^{2}}+C[\tau] \frac{3}{\vartheta \Delta \tau}+K(\tau)\right] \cdot \Delta u(\vartheta \Delta \tau)=m\left[\frac{6}{\vartheta \Delta \tau} \dot{u}(\tau)+3 \ddot{u}(\tau)\right]+} \\
+C(\tau)\left[3 \dot{u}(\tau)+\frac{\vartheta \Delta \tau}{2} \ddot{u}(\tau)\right]+\Delta f(\vartheta \Delta \tau)-N e(\tau),
\end{gathered}
$$

which is used instead of the equation (10).

Algorithm implementation of the Wilson's method is as follows.

1. The initial velocity $\dot{u}(\tau)$ and displacement $u(\tau)$ are given by the initial conditions of the problem, or are known from the previous integration steps.

2. With these values and using a right differences the partial derivatives are calculated. For Example,

$$
\frac{\partial \bar{R}_{x}}{\partial \chi}=\frac{\bar{R}_{x}(\chi+\Delta \chi, \delta, E, G)-\bar{R}_{x}(\chi, \delta, E, G)}{\Delta \chi},
$$

where $\Delta \chi(\Delta \delta, \Delta E, \Delta G)$ is advisable to take equal 0.0001 .

3. Residuals are calculated for each variable

$$
N e(\tau)=m \ddot{u}+P(u, \dot{u})-f(\tau) .
$$

4. The increment of function is calculated $f(\tau)$

$$
\Delta f(\tau)=f(\tau+\vartheta \Delta \tau)-f(\tau)
$$

and the right-hand side of equation (18):

$$
\Delta f(\tau)-N e(\tau)
$$

5. By the formulas (14), (10), (11) at time $\tau=\tau+\vartheta \Delta \tau$ we define the increments of coordinate of the journal center, velocities and accelerations

$$
\Delta x(\vartheta \Delta \tau), \Delta y(\vartheta \Delta \tau), \Delta \dot{x}(\vartheta \Delta \tau), \Delta \dot{y}(\vartheta \Delta \tau), \Delta \ddot{x}(\vartheta \Delta \tau), \Delta \ddot{y}(\vartheta \Delta \tau) .
$$

Then the result is recalculated for the interval $\Delta \tau$ by the formulas (7), (8), (9):

$$
\begin{gathered}
\Delta \ddot{u}(\Delta \tau)=\frac{1}{\vartheta} \Delta \ddot{u}(\vartheta \Delta \tau) ; \quad \Delta \dot{u}(\Delta \tau)=\Delta \tau \ddot{u}(\tau)+\frac{\Delta \tau}{2 \vartheta} \Delta \ddot{u}(\vartheta \Delta \tau) ; \\
\Delta u(\Delta \tau)=\Delta \tau \dot{u}(\tau)+\frac{\Delta \tau^{2}}{2} \ddot{u}(\tau)+\frac{\Delta \tau^{2}}{6 \vartheta} \Delta u(\vartheta \Delta \tau) .
\end{gathered}
$$


6. Accelerations, velocities and coordinates of the journal center in the end of the time interval $\Delta \tau$ are determined as:

$$
\begin{aligned}
& \ddot{u}(\tau+\Delta \tau)=\ddot{u}(\tau)+\Delta \ddot{u}(\Delta \tau) ; \\
& \dot{u}(\tau+\Delta \tau)=\dot{u}(\tau)+\Delta \dot{u}(\Delta \tau) ; \\
& u(\tau+\Delta \tau)=u(\tau)+\Delta u(\Delta \tau) .
\end{aligned}
$$

Optimum performance of stability and accuracy are achieved by varying the parameter $\vartheta$. The authors of [10] showed that the unconditional stability of the method is achieved by $\vartheta=1.37$.

For tasks of nonlinear oscillations of the journal on the lubricating layer of the bearing the parameter $\vartheta$ can be changed, as shown by our study, from 1.0 to 1.8 . And at $\vartheta=1.37$ the integration time is the smallest. The correction by the magnitude of the discrepancy $\mathrm{Ne}(\tau)$ is similar changes the solution step on time. Therefore, the implemented algorithm, the value is taken to be 1.37, and the step of calculating adjusted to the minimum value of the discrepancy:

if $\max _{u}|N e(\tau)|>\varepsilon_{1}, \Delta \tau=\Delta \tau \cdot 0.75$, if $\max _{u}|N e(\tau)|<\varepsilon_{2}, \Delta \tau=\Delta \tau \cdot 1.5$,

where $\varepsilon_{1}=0.0001$ and $\varepsilon_{2}=0.00001$ are defined by numerical experiments.

These algorithms have been successfully used in solving tasks of the dynamics of heavy-loaded units of the internal combustion engine $[11,12]$.

This work was supported by a grant of the Ministry of Education and Science of the Russian Federation for applied research, code 2014-14-579-0109. The unique identifier for Applied Scientific Research (project) is RFMEFI57714X0102. Agreement no. 14.577.21.0102.

\section{References}

1. Burgvits A.G., Zavyalov G.A. [Stability of Motion Shaft Bearings Fluid Friction]. Moscow, Mashinostroenie Publ., 1964. (in Russian)

2. Kurin L.M. [Development of Algorithms and Software Linear Analysis of Flexural Vibrations of Rotors of Turbomachines Taking into Account Distortions in the Necks of Bearings]. Dis. ... kand. tekhn. nauk, Chelyabinsk, 1989. (in Russian)

3. Kirk R.G., Gunter E.J. Short Bearing Analysis Applied to Rotor Dynamics. Part 1: Theory. Journal of Lubrication Technology. Series F. Problems of Friction and Lubricant, 1976, no. 1, pp. 47-56.

4. Prokop'ev V.N. [Applied Theory and Methods of Calculation of Hydrodynamic HeavyLoaded Bearings]. Dis. ... dokt. tekhn. nauk, Chelyabinsk, 1985. (in Russian)

5. Prokop'ev V.N, Boyarshinova A.K., Gavrilov K.V. Hydromechanical Characteristics of Heavy-Loaded Bearings with the Non-Circular Journal and Bearing. Problems of Mechanical Engineering and Reliability of the Machines, 2009, no. 4, pp. 98-104. 
6. Zadorozhnaya E.A., Levanov I.G., Pyryev S.A. Calculation of Efficiency Tribo-Unit "Gudgeon Pin - the Little End" of Tractor Diesel. Journal of Mechanical Engineering, 2013, no. 12 , pp. $42-47$.

7. Rakitsky Yu, Ustinov S.M., Chernorutsky N.G. [Numerical Methods for Solving Rigid Systems]. Moscow. Nauka Publ., 1979. (in Russian)

8. Clough R.V., Penzien J. [The dynamics of structures]. Moscow. Stroyizdat Publ., 1979. (in Russian)

9. Tabarny V.G., Vasinyuk V.E., Kolyada Y.V. Some Numerical Integration Methods and Their Application to the Analysis of Nonlinear Circuits Machine. Theoretical Electrical Engineering, 1972, vol. 14, pp. 57-64.

10. Volmir A.S., Kuranov B.A., Turbaivsky A.T. [Statics and dynamics of complex structures]. Moscow. Mashinostroenie Publ., 1989. (in Russian)

11. Surkin V.I., Zadorozhnaya E.A., Niyazov H.M. Calculation Heavy-Loaded Tribo-Units of High Performance Diesel Engine. Bulletin of the South Ural State University. Series "Engineering", 2012, no. 12(271), pp. 97-102.

Yuriy V. Rozhdestvenskiy, Doctor of Technical Sciences, Professor, Academician of the Russian Academy of Transport, Dean of Faculty of Automobile and Tractor Engineering, South Ural State University, Chelyabinsk, Russian Federation, ruv@susu.ac.ru.

Elena A. Zadorozhnaya, Doctor of Technical Sciences, Associate Professor, Department of Motor Transports and Service of Automobiles, South Ural State University, Chelyabinsk, Russian Federation, elena-nea@rambler.ru.

Roman O. Chuchelov, Undergraduate, Department of Motor Transports and Service of Automobiles, South Ural State University, Chelyabinsk, Russian Federation, chro_174@mail.ru.

Dmitriy I. Raskatov, Undergraduate, Department of Motor Transports and Service of Automobiles, South Ural State University, Chelyabinsk, Russian Federation, dir.77@mail.ru. 